\title{
Testing Theories of Post-Error Slowing
}

\author{
Gilles Dutilh ${ }^{1}$, Joachim Vandekerckhove ${ }^{2}$, Birte U. Forstmann ${ }^{1}$, Emmanuel Keuleers ${ }^{3}$, Marc Brysbaert ${ }^{3}$, \\ and Eric-Jan Wagenmakers ${ }^{1}$ \\ ${ }^{1}$ University of Amsterdam, The Netherlands \\ ${ }^{2}$ University of Leuven, Belgium \\ ${ }^{3}$ Ghent University, Belgium
}

\author{
Correspondence concerning this article should be addressed to: \\ Gilles Dutilh \\ University of Amsterdam, Department of Psychology \\ Roetersstraat 15 \\ 1018 WB Amsterdam, The Netherlands \\ Ph: $(+31)$ 20-525-6629 \\ E-mail may be sent to gilles.dutilh@gmail.com.
}

\begin{abstract}
People tend to slow down after they make an error. This phenomenon, generally referred to as post-error slowing, has been hypothesized to reflect perceptual distraction, time wasted on irrelevant processes, a priori bias against the response made in error, increased variability in a priori bias, or an increase in response caution. Although the response caution interpretation has dominated the empirical literature, little research has attempted to test this interpretation in the context of a formal process model. Here we used the drift diffusion model to isolate and identify the psychological processes responsible for post-error slowing. In a lexical decision data set comprised of $1,094,886$ responses we found that post-error slowing was associated with an increase in response caution and - to a lesser extent-a change in response bias. In the present data set, we found no evidence that post-error slowing is caused by perceptual distraction or time wasted on irrelevant processes. These results support a response monitoring account of post-error slowing.
\end{abstract}

Keywords: Response caution, Response time distributions, Diffusion model decomposition, Lexical decision.

What does a man do after he makes an error? ${ }^{1}$ This question is just a valid as when it was first articulated by Rabbitt and Rodgers (1977) over 30 years ago. One answer to this question is that, after he has made an erroneous decision, a man slows down on his next decision-an empirical regularity known as post-error slowing (PES; Laming, 1968, 1979b, 1979a; Rabbitt, 1966, 1979; Rabbitt \& Rodgers, 1977). However, this answer raises a new and more interesting question, namely, why does a man slow down after he makes an error? Various answers have been proposed and one of the main goals of this article is to implement these answers in a formal model of decision making so as to compare their adequacy in a precise and quantitative fashion.

The competing explanations for PES, detailed in the next section, are (1) increased response caution; (2) a priori bias away from the response that was just made in error; (3) an overall decrease in the across-trial variability of a priori bias; (4) distraction of attention; (5) delayed startup due to irrelevant processes (e.g., overcoming disappointment). We propose that these five explanations map on uniquely to parameters in a drift diffusion model for response time (RT) and ac- curacy (Ratcliff, 1978; Ratcliff \& McKoon, 2008). This oneto-one mapping between psychological processes and model parameters allows an informative diffusion model decomposition of PES and a rigorous assessment of the extent to which each explanation (or indeed any combination of them) holds true.

A major practical obstacle that we needed to overcome is that the drift diffusion model requires relatively many observations to produce informative parameter estimates; as a rule of thumb, the model requires at least 10 error RTs in each experimental condition. Because the interest here centers on trials that follow an error, this means that the model requires at least 10 errors that immediately follow an error. With an error rate of 5\% throughout, the minimum number of observations is already 4,000. Thus, a reliable diffusion model decomposition of PES requires a relatively large data set. Here we fit the model to a lexical decision data set featuring 39 participants who each completed 28,074 trials for a

\footnotetext{
${ }^{1}$ For historical reasons we use the word "man" instead of "person", even though the latter is slightly more accurate and much more politically correct.
} 
grand total of $1,094,886$ trials (Keuleers, Brysbaert, \& New, 2010).

In the next sections we briefly discuss the different explanations for PES and formalize these predictions in the context of the drift diffusion model. We then test the different explanations by fitting the model to the lexical decision data from Keuleers, Brysbaert, and New (2010). To the best of our knowledge, this is the second time that competing explanations for PES are tested in the framework of a formal model of decision making. The first study, due to White, Ratcliff, Vasey, and McKoon (2010b), also presented a diffusion model decomposition of PES - the differences and similarities between our study and that of White et al. (2010b) are analyzed in the final section.

\section{Explanations for Post-Error Slowing}

Over the years, several explanations have been proposed to account for PES. The first explanation (i.e., increased response caution) is that an error prompts people to accumulate more information before they initiate a decision. The underlying idea is that people can adaptively change their response thresholds-becoming slightly less cautious after a correct response, and more cautious after an error-and thereby self-regulate to an optimal state of homeostasis characterized by fast responses and few errors (e.g., Botvinick, Braver, Barch, Carter, \& Cohen, 2001; Cohen, Botvinick, \& Carter, 2000; Brewer \& Smith, 1989; Fitts, 1966; Rabbitt \& Rodgers, 1977; Smith \& Brewer, 1995; Vickers \& Lee, 1998). This explanation is so appealing that it is often assumed to be correct without further testing. That is, PES is often interpreted as a direct measure of cognitive control. Conclusions about cognitive control are then based on associations between PES and physiological measures such as anterior cingulate activity (Li, Huang, Constable, \& Sinha, 2006; Danielmeier, Eichele, Forstmann, Tittgemeyer, \& Ullsperger, 2011), error-related negativity (ERN) and positivity (Pe, Hajcak, McDonald, \& Simons, 2003), or cortisol levels (Tops \& Boksem, 2010). Alternatively, conclusions about cognitive control may be based on a comparison of PES between clinical groups (e.g., Shiels \& Hawk, 2010).

The second explanation (i.e., a priori bias) is that people become negatively biased against the response option that was just executed in error (e.g., Laming, 1968, 1979b; Rabbitt \& Rodgers, 1977). This implies that errors facilitate response alternations and hinder response repetitions, both with respect to response speed and probability of occurrence.

The third explanation (i.e., decreased variability in bias) is that, following an error, people might wait a little longer before they start to accumulate stimulus-related information. The idea, first promoted by Laming $(1968,1979 a)$, is that in speeded RT tasks people often start to sample information from the display even before the stimulus is presented. This advance sampling of stimulus-unrelated information induces trial-to-trial variability in a priori bias. This variability may cause fast errors, and therefore a cautious participant starts the evidence accumulation process only after stimulus onset.
The fourth explanation (i.e., distraction of attention) is that the occurrence of an error is an infrequent, surprising event that distracts participants during the processing of the subsequent stimulus (Notebaert et al., 2009). Thus, the errorinduced distraction contaminates the process of evidence accumulation.

The fifth explanation (i.e., delayed startup) is that errors delay the start of evidence accumulation on the next trialfor instance, participants might need time after an error to re-assess their own performance level and to overcome disappointment (Rabbitt \& Rodgers, 1977).

In the literature, the first explanation of PES (i.e., increased response caution) has always been the most dominant. However, Rabbitt (1966, p. 272) already concluded that "the present data do not allow a choice between possible explanations". Other studies did not test the competing explanations in a rigorous and quantitative manner (but see White et al., 2010b). Here we set out to test the above five explanations in the context of what is arguably the most popular and successful model for response times and accuracy, the drift diffusion model (Ratcliff, 1978; Ratcliff \& McKoon, 2008).

\section{A Drift Diffusion Model Decomposition of Response Times}

In the analysis of speeded two-choice tasks, performance is usually summarized by mean RT and proportion correct. Although concise, this summary ignores important aspects of the data and makes it difficult to draw conclusions about the underlying cognitive processes that drive performance (Wagenmakers, Van der Maas, \& Grasman, 2007). A more detailed and more informative analysis takes into account the entire RT distributions for both correct and error responses, in addition to proportion correct. These RT distributions can be analyzed with the help of formal models - here we focus on the drift diffusion model.

The drift diffusion model has been successfully applied to a wide range of experimental tasks including brightness discrimination, letter identification, lexical decision, recognition memory, signal detection and the implicit association test (e.g., Ratcliff, 1978; Ratcliff, Gomez, \& McKoon, 2004; Ratcliff, Thapar, \& McKoon, 2006; Wagenmakers, Ratcliff, Gomez, \& McKoon, 2008; Dutilh, Vandekerckhove, Tuerlinckx, \& Wagenmakers, 2009; Ratcliff, Thapar, \& McKoon, 2010; Klauer, Voss, Schmitz, \& Teige-Mocigemba, 2007; Van Ravenzwaaij, Van der Maas, \& Wagenmakers, in press). In these tasks and others, the model has been used to decompose the behavioral effects of phenomena such as practice (Dutilh et al., 2009; Dutilh, Krypotos, \& Wagenmakers, in press; Petrov, Horn, \& Ratcliff, in press), aging (Ratcliff, Thapar, \& McKoon, 2001; Ratcliff et al., 2006, 2010), psychological disorders (White, Ratcliff, Vasey, \& McKoon, 2009, 2010a; White et al., 2010b), sleep deprivation (Ratcliff \& Van Dongen, 2009), intelligence (Ratcliff, Schmiedek, \& McKoon, 2008; Schmiedek, Oberauer, Wilhelm, Suss, \& Wittmann, 2007; Van Ravenzwaaij, Brown, \& Wagenmak- 
ers, in press), and so forth.

The success of the drift diffusion model is due to several factors. First, the drift diffusion model takes into account not just mean RT but considers entire RT distributions for correct and error responses; second, the drift diffusion model generally provides an excellent fit to observed data with relatively few parameters free to vary; third, the drift diffusion model accounts for all current benchmark phenomena (Brown \& Heathcote, 2008); fourth, the model allows researchers to decompose observed performance into constituent cognitive processes of interest; fifth, the model has passed several tests of selective influence and this increases one's confidence that the model parameters represent the intended cognitive process (Voss, Rothermund, \& Voss, 2004; Ratcliff \& Rouder, 1998; Wagenmakers et al., 2008); finally, the drift diffusion model has been linked to the dynamics of neural firing rates, providing some insight as to how a diffusion process might be implemented in the brain (Gold \& Shadlen, 2007). Additional advantages (and limitations) of a diffusion model analysis are discussed in more detail in Wagenmakers (2009).

Here we briefly introduce the drift diffusion model as it applies to the lexical decision task, a task where participants have to decide quickly whether a presented letter string is a word (e.g., party) or a nonword (e.g., drapa). The core of the model is the Wiener diffusion process that describes how the relative evidence for one of two response alternatives accumulates over time. The meandering lines in Figure 1 illustrate the continuous accumulation of noisy evidence following the presentation of a word stimulus. When the amount of diagnostic evidence for one of the response options reaches a predetermined response threshold (i.e., one of the horizontal boundaries in Figure 1), the corresponding response is initiated. The dark line in Figure 1 shows how the noise inherent in the accumulation process can sometimes cause the process to end up at the wrong (i.e., nonword) response boundary.

The standard version of the drift diffusion model decomposes RTs and proportion correct in seven different parameters:

1. Mean drift rate $(v)$. Drift rate quantifies rate of information-accumulation from the stimulus. This means that when the absolute value of drift rate is high, decisions are fast and accurate; thus, $v$ relates to task difficulty or subject ability.

2. Across-trial variability in drift rate $(\eta)$. This parameter reflects the fact that drift rate may fluctuate from one trial to the next, according to a normal distribution with mean $v$ and standard deviation $\eta$. The parameter $\eta$ allows the drift diffusion model to account for data in which error responses are systematically slower than correct responses (Ratcliff, 1978).

3. Boundary separation $(a)$. Boundary separation quantifies response caution and modulates the speed-accuracy tradeoff: At the price of an increase in RT, participants can decrease their error rate by widening the boundary separation (e.g., Forstmann et al., 2008).

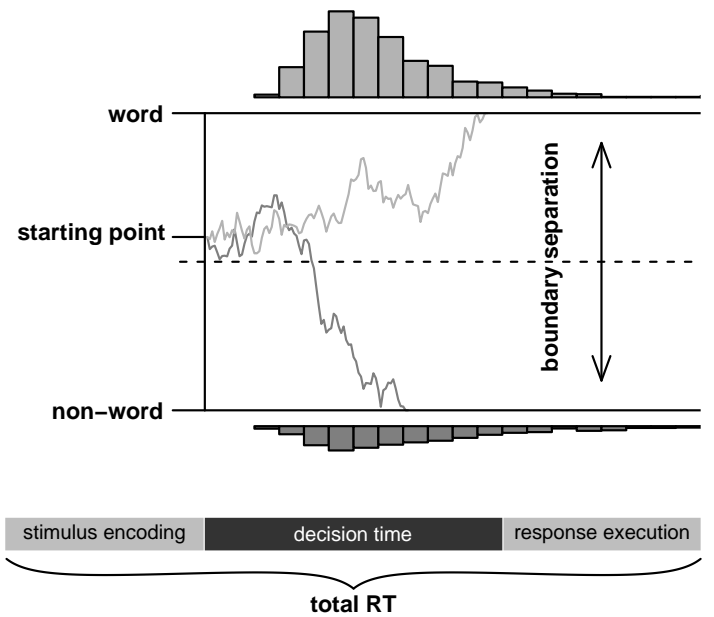

Figure 1. The drift diffusion model as it applies to the lexical decision task. A word stimulus is presented (not shown) and two example sample paths represent the accumulation of evidence which result in one correct response (light line) and one error response (dark line). Repeated application of the diffusion process yields histograms of both correct responses (upper histogram) and incorrect responses (lower histogram). As is evident from the histograms, the correct, upper word boundary is reached more often than the incorrect, lower nonword boundary. The total RT consists of the sum of a decision component, modeled by the noisy accumulation of evidence, and a non-decision component that represents the time needed for processes such as stimulus encoding and response execution.

4. Mean starting point $(z)$. Starting point reflects the $a$ priori bias of a participant for one or the other response. This parameter is usually manipulated via payoff or proportion manipulations (Edwards, 1965; Wagenmakers et al., 2008; but see Diederich \& Busemeyer, 2006). Often, $z$ is reported as a proportion of boundary separation $a$ and referred to as bias $B$.

5. Across-trial variability in starting point $\left(s_{z}\right)$. This parameter reflects the fact that starting point may fluctuate from one trial to the next, according to a uniform distribution with mean $z$ and range $s_{z}$. The parameter $s_{z}$ also allows the drift diffusion model to account for data in which error responses are systematically faster than correct responses. Analogous to the transformation of $z$ to $B, s_{z}$ is often transformed to $s_{B}$.

6. Mean of the non-decision component of processing $\left(T_{e r}\right)$. This parameter encompasses the time spent on common processes, i.e., processes executed irrespective of the decision process. The drift diffusion model assumes that the observed RT is the sum of the non-decision component and the decision component (Luce, 1986):

$$
R T=D T+T_{e r},
$$


where DT denotes decision time. Therefore, nondecision time $T_{e r}$ does not affect response choice and acts solely to shift the entire RT distribution.

7. Across-trial variability in the non-decision component of processing $\left(s_{t}\right)$. This parameter reflects the fact that non-decision time may fluctuate from one trial to the next, according to a uniform distribution with mean $T_{e r}$ and range $s_{t}$. The parameter $s_{t}$ also allows the model to capture RT distributions that show a relatively shallow rise in the leading edge.

\section{From Process to Parameter: A Drift Diffusion Model Perspective on Post-Error Slowing}

Many recent applications of the drift diffusion model have been exploratory in nature; for instance, researchers have used the drift diffusion model to study the psychological processes that change with practice (Dutilh et al., 2009, in press), sleep-deprivation (Ratcliff \& Van Dongen, 2009), hypoglycemia (Geddes et al., in press), and dysphoria (White et al., 2009, 2010a), but this work was seldom guided by strong prior expectations and theories. This is different in the case of PES, perhaps because explanations for PES have originated in part from a framework of sequential information processing (e.g., Laming, 1979a). Therefore, the competing explanations for PES - in terms of the cognitive processes that change after an error-can be mapped selectively to different parameters in the drift diffusion model, as is shown in Figure 2.

Thus, the cognitive process explanation of increased response caution maps onto an increase in boundary separation $a$; the explanation of a priori bias corresponds to a shift in starting point $z$ away from the boundary that was just reached in error $^{2}$; the explanation of decreased variability in bias translates to a decrease in across-trial variability $s_{z}$; the explanation of distraction of attention entails a decrease in mean drift rate $v$; and, finally, the explanation of delayed startup is associated with an increase in mean non-decision time $T_{e r}$. The unique link between process and parameter means that competing explanations for PES can be rigorously tested in any particular paradigm, as long as the drift diffusion model applies and the data set is sufficiently large. In the context of PES, the latter concern is particularly acute.

\section{Method}

The present data set was originally collected to validate a new measure for word frequency (i.e., SUBTLEX-NL; Keuleers, Brysbaert, \& New, 2010). Each of 39 participants contributed 28,074 lexical decisions for a grand total of $1,094,886$ decisions. Half of the stimuli were uniquely presented words and the other half were uniquely presented nonwords. The word stimuli were selected from the CELEX database (Baayen, Piepenbrock, \& Van Rijn, 1993) and the nonword stimuli were created with the Wuggy pseudoword generator (Keuleers \& Brysbaert, 2010).
The experiment was presented in blocks of 500 trials with a self-paced break after every 100 trials. Each trial started with a $500 \mathrm{~ms}$ fixation period. The stimulus was then presented until the participant responded, up to a maximum of $2000 \mathrm{~ms}$. A new trial started $500 \mathrm{~ms}$ after the response. Participants received feedback about their accuracy after each block of 500 trials. Importantly, participants did not receive trial-by-trial feedback concerning errors. This means that any post-error effects are not contaminated by the possibly distracting presence of error feedback. A more detailed description of the experimental methods is presented in Keuleers, Brysbaert, and New (2010) and Keuleers, Diependaele, and Brysbaert (2010).

The enormous amount of lexical decision trials in this data set contains a commensurate amount of errors; across all participants, 118,566 trials (i.e., $10.80 \%$ ) were made in error. This abundance of errors allowed us to examine the explanations for PES across various conditions. Specifically, we were able to compare post-error effects separately for nonword stimuli and for word stimuli of varying word frequencies. ${ }^{3}$ That is, we used word frequency (based on SUBTLEX) to divide all words into six equally large bins, the five cut points being $0.11,0.48,1.33,3.73$, and 14.16 occurrences per million.

\section{Results}

Below we first discuss the effects of errors on observed performance, that is, RT and proportion correct. Next, we fit the drift diffusion model to the data and discuss the effects of errors on the latent psychological processes hypothesized to explain PES. ${ }^{4}$

\section{Post-Error Effects on Observed Data}

The different hypotheses about PES entail effects on RT, effects on proportion correct, or a combination of the two. It is therefore informative to show-both for posterror trials and post-correct trials, and for different stimulus categories-entire distributions of RT for correct and error responses, together with proportion correct. A convenient tool to paint this multivariate picture is the quantile probability plot (e.g., Ratcliff, 2002). Figure 3 shows a quantile probability plot for the data from Keuleers, Brysbaert, and New (2010), based on averaging RT quantiles and proportions across individual participants.

Figure 3 features two important factors in the design of this study, that is, post-error trials vs. post-correct trials (i.e., triangles vs. circles) and word frequency of the current stimulus (including nonwords, grey scales). The plot is

\footnotetext{
${ }^{2}$ In the results section we report bias $B$ instead of $z$ and $s_{B}$ instead of $s_{z}$.

${ }^{3}$ We also investigated the difference between response repetitions and alternations, as we will explain later on.

${ }^{4}$ The analyses reported here concern the difference between post-correct trials and post-error trials. Results based on the difference between pre-error and post-error trials yielded quantitatively and qualitatively similar results. These results can be found on the first author's website.
} 


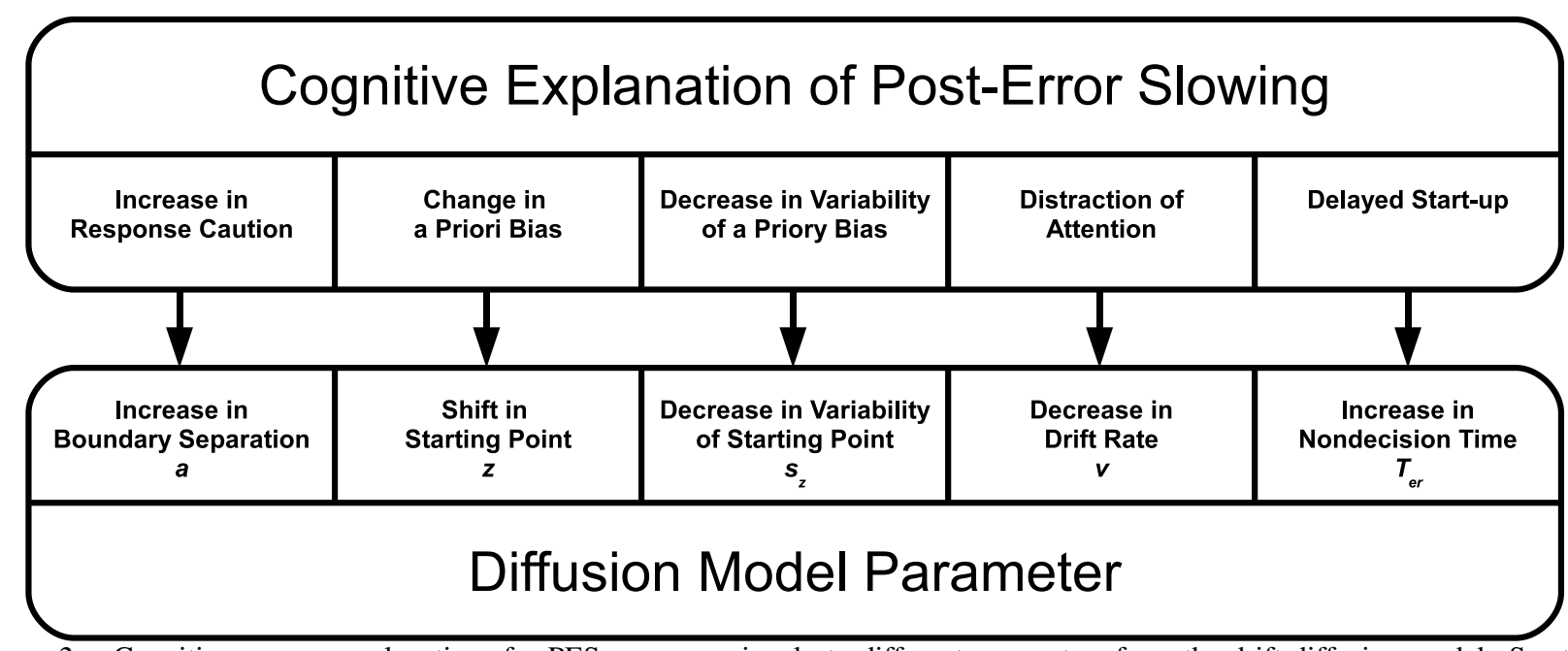

Figure 2. Cognitive process explanations for PES map on uniquely to different parameters from the drift diffusion model. See text for details.

read as follows. Each column of points summarizes a single RT distribution by five quantiles (i.e., the $.1, .3, .5, .7$, and .9 quantiles - the .1 quantile, for instance, is the RT value for which $10 \%$ of the RT distribution is faster; the .5 quantile is the median RT). Each column in the right half of the figure describes a correct RT distribution for a particular condition; its position on the $x$-axis shows the corresponding proportion correct (e.g., $x=0.61$ for the post-correct, low-frequency "freq 1" words). This correct RT distribution has an associated distribution of incorrect RTs, shown in the left half of the figure (e.g., $x=1-0.61=0.39$ for the post-correct, low-frequency "freq 1" words).

Figure 3 shows that word frequency benefits performance: high frequency words are associated with low error rates and fast RT quantiles. More important for the present study, RT quantiles are slower after an error (triangles) than after a correct response (circles). The slowdown is smallest in the leading edge of the distribution (for correct responses, on average $3 \mathrm{~ms}$ at the .1 quantile) and biggest at the tail (on average $38 \mathrm{~ms}$ at the .9 quantile). These PES effects are more pronounced for low frequency words (frequency groups one and two) than for high frequency words. Figure 4 zooms into the PES effect by presenting the data (and the model fit discussed later) as a delta plot (De Jong, Liang, \& Lauber, 1994; Pratte, Rouder, Morey, \& Feng, 2010; Speckman, Rouder, Morey, $\&$ Pratte, 2008). In a delta plot, the factor of interest-in this case, the PES effect-is shown as a function of response speed. Here, Figure 4 shows the average PES effect (i.e., the PES effect across all experimental conditions, quantileaveraged across participants). The delta plot indicates that the PES effect is negligible for the very fast responses and becomes more prominent when response times are slow.

For the low frequency words, accuracy is slightly higher following an error than following a correct response. This difference of about $2.4 \%$ is supported by a default Bayesian paired samples t-test (Rouder, Speckman, Sun, Morey, \& Iverson, 2009; Wetzels, Raaijmakers, Jakab, \& Wagenmakers, 2009; Wetzels et al., in press), as the Bayes factor against the null hypothesis is 52 ; this indicates that the data are 52 times more likely under the alternative hypothesis than under the null hypothesis. The small decrease in post-error accuracy for nonwords (about .7\%) is supported by a Bayes factor of 10 .

Although the post-error effects in Figure 3 are qualitatively consistent across different levels of word frequency, it is not direct how they should be interpreted in terms of underlying psychological processes. The data seem to support an explanation in terms of increased response caution. However, attentional effects could also play a role. To understand the underlying, possibly interacting processes that cause the post-error slowing effect, we now turn to a diffusion model decomposition.

\section{Post-Error Effects on Latent Processes}

We fit the model to the individual data using the MATLAB package "DMAT" (Vandekerckhove \& Tuerlinckx, 2007, 2008). As noted above, the size of the present data set allowed us to examine several experimental conditions or factors. The primary factor was the correctness of the previous trial, and secondary factors were stimulus type (i.e., word vs. nonword) on the current trial, word frequency of the word stimuli on the current trial, and stimulus type on the previous trial.

For the secondary factors we used the BIC (Bayesian information criterion; Schwarz, 1978; Raftery, 1995) to eliminate excess parameters and select the most parsimonious model that still gives an acceptable fit to the data. In this BIC-best model the different factors affected the model's parameters as follows: Stimulus type of the current trial was 


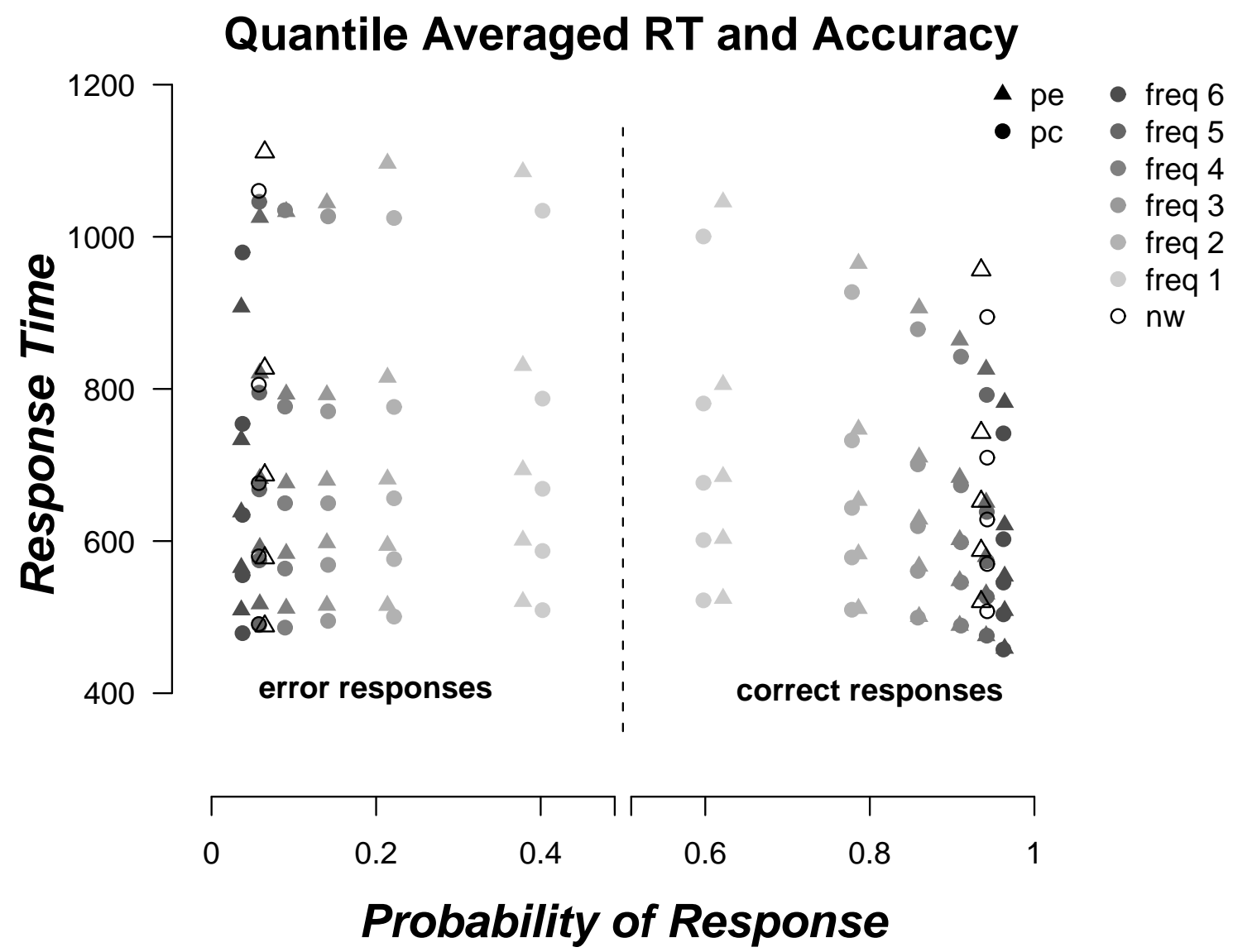

Figure 3. Post-error trials are slower and somewhat more accurate than post-correct trials. This pattern holds for all word stimuli but it is more pronounced for low frequency words ("freq 1") than for high frequency words ("freq 6"). In addition, the effect is more pronounced in the tail of the RT distribution. NB. In this quantile probability plot, the $y$-axis indicates five RT quantiles that together summarize the entire RT distribution. The $x$-axis indicates proportion correct; hence, each condition yields two RT distributions, one for correct responses, shown on the right, and one for error responses, shown on the left.

allowed to affect drift rate $v$ and its variability $\eta$, and nondecision time $T_{e r}$. Word frequency was allowed to affect drift rate $v$ and $T_{e r}$. Stimulus type of the previous trial was allowed to affect bias $B$ and its variability $s_{B}$. This BIC-best model was then used to quantify the impact of the primary factor of interest, that is, the factor post-error vs. post-correct was allowed to affect all of the diffusion model parameters.

Figure 4, discussed earlier, compares the data against the model predictions. The solid dots represent the empirical data (i.e., the PES effect in all experimental conditions, quantile-averaged across participants), and the lines with open dots represent the predictions of the best-fitting model parameters. Overall, the fit is good, except perhaps for the .9 quantile; this may be due to the fact that this quantile is the most difficult to estimate reliably.

Figure 5 and Figure 7 show the estimates for the diffusion model parameters, averaged over participants. The as- sociated Figures 6 and 8 present the differences in the model parameters for post-correct vs. post-error trials. The most obvious effect in Figure 5 and 6 is the increase of boundary separation after an error, shown in the upper left panel. This increase in boundary separation indicates that on average, participants become more cautious after committing an error. The Rouder et al. (2009) default Bayesian t-test indicates that the data are about 180,000 times more likely under the alternative hypothesis of unequal boundary separation than under the null hypothesis of equal boundary separation; this is considered extreme evidence in favor of an effect.

The post-error effect on bias (bottom left panel) is more complicated because it was also affected by the stimulus type of the previous trial. The figure suggests that, after an erroneous response, whether this was "word" or "nonword", participants are on average more likely to respond "word" on the next trial. However, a stronger effect seems to be that partic- 


\section{word frequency group 1}



word frequency group 4



word frequency group 2



word frequency group 5



nonwords

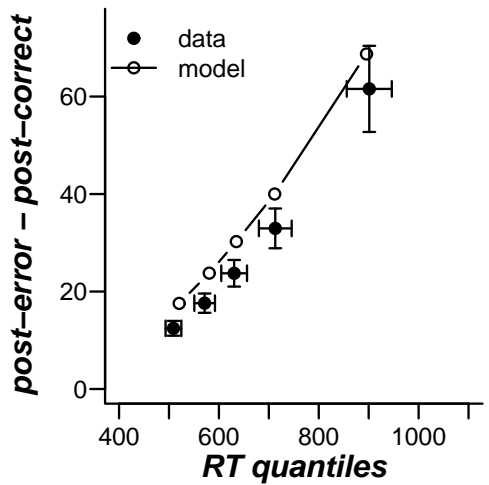

word frequency group 3

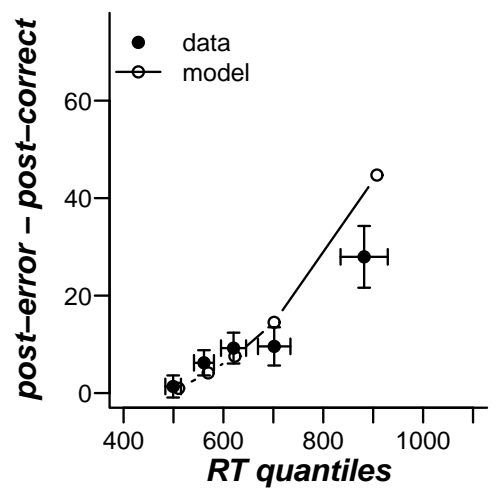

word frequency group 6

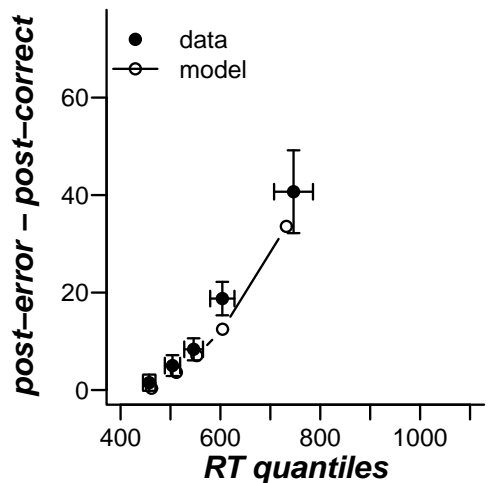

Figure 4. Delta plots of PES effect against response speed in post-correct trials, separately for all word frequencies and nonwords. Solid circles represent empirical data (error bars indicate standard errors) and lines with open circles represent predictions from the model. For both empirical data and model predictions, the effects are obtained by quantile-averaging the results across participants.

ipants have a preference for giving the same response as the one given on the previous trial, regardless of the correctness of that trial, i.e., a response repetition effect.

The right two panels show the effects on drift rate and non-decision time. Neither drift rate nor non-decision time show any effect of the correctness of the previous trial. However, with word frequency, drift rate increases and non- decision time decreases.

Figure 7 shows the estimates for the variability parameters of the diffusion model, averaged over participants. The associated Figure 8 presents the differences in the model parameters for post-correct vs. post-error trials. The figures suggest that none of the variability parameters are responsible for PES. However, we did find that the variability in drift 



Figure 5. The four main parameters of the diffusion model shown separately for post-correct and post-error trials. Bias $B$ was estimated separately for post-word and post-nonword conditions. Drift rate $v$ and non-decision time $T_{e r}$ were estimated separately for nonwords and for different categories of word frequency. The most prominent post-error effect is an increase in boundary separation. Error bars represent standard errors of the mean.

$\eta$ is larger for words than it is for nonwords, replicating an earlier lexical decision study (Dutilh et al., 2009).

In sum, the diffusion model decomposition supports an explanation of PES in terms of increased response caution.

\section{Concluding Comments}

What does a man do after he makes an error? Data from a 1,094,886- trial lexical decision task showed that people slow down after an error, and a diffusion model decomposition showed that this slowdown can be attributed almost exclusively to an increase in response caution. This result confirms the traditional explanation of PES in terms of selfregulation and cognitive control (e.g., Botvinick et al., 2001; Cohen et al., 2000; Brewer \& Smith, 1989; Fitts, 1966; Hajcak et al., 2003; Li et al., 2006; Rabbitt \& Rodgers, 1977; Shiels \& Hawk, 2010; Smith \& Brewer, 1995; Tops \& Bok- sem, 2010; Verguts, Notebaert, Kunde, \& Wühr, 2011; Vickers \& Lee, 1998): that is, people adaptively change their response thresholds to a possibly nonstationary environmentby becoming more daring after each correct response, and by becoming more cautious after each error, people reach an optimal state of homeostasis that is characterized by fast responses and few errors.

Although this explanation of PES has strong face validity it is entirely possible that other explanations could also be correct in particular cases. Only by applying a formal process model can we evaluate the competing accounts of PES quantitatively. Our results are partially consistent with those of White et al. (2010b), who applied the drift diffusion model to data from a recognition memory task and found that participants with high-trait anxiety responded more carefully after making an error (i.e., increased boundary separa- 

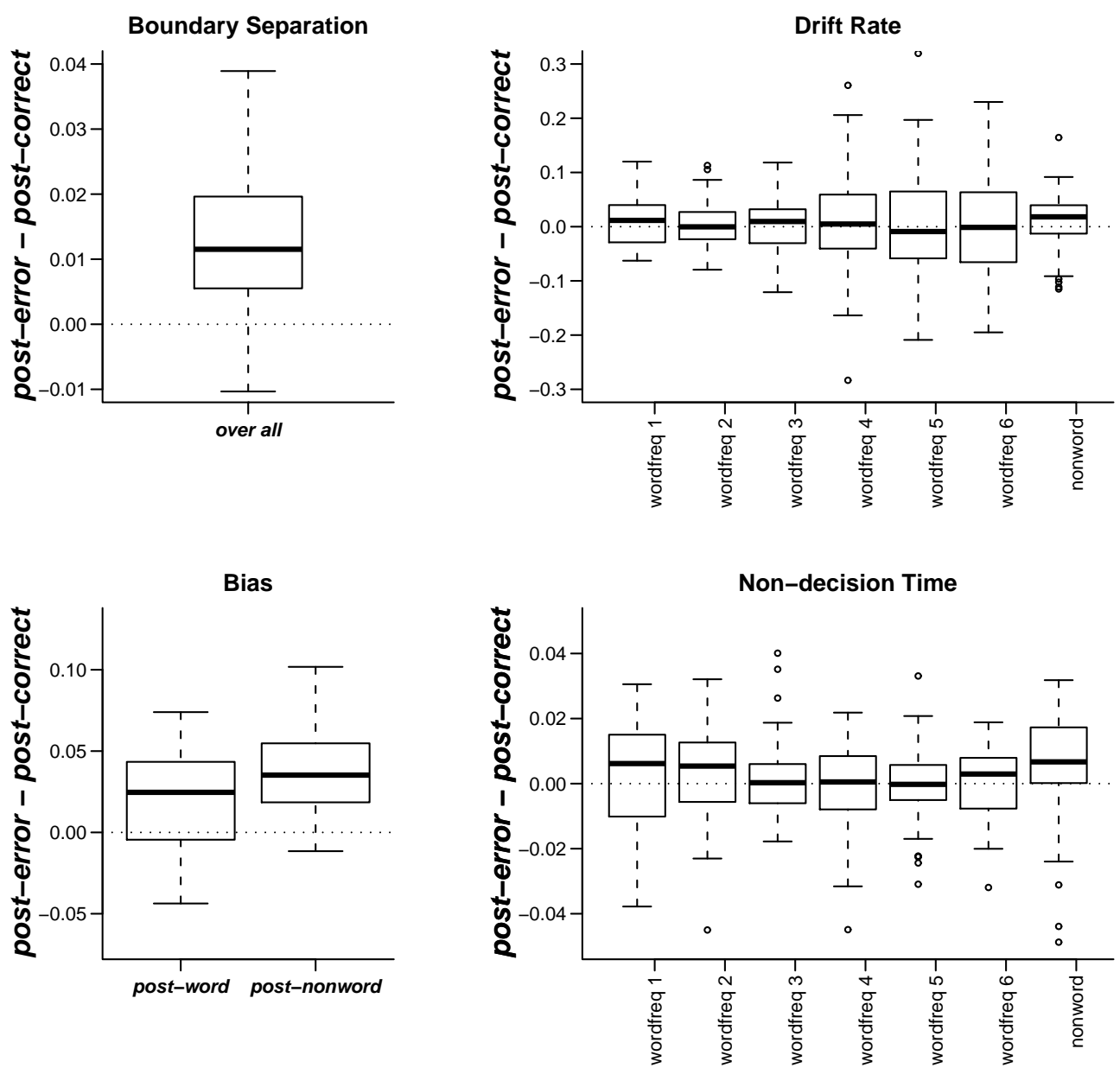

Figure 6. For each of the four main diffusion model parameters, box plots represent the distribution of the PES effect over participants. Comparison with the dashed horizontal line at zero suggests PES effects express themselves only on boundary separation and a priori bias (after an erroneous nonword response). Boxes contain $50 \%$ of the values, whiskers enclose $80 \%$ of the values.

tion $a$ following an error). However, the data from White et al. (2010b) did not show a response caution effect for participants with low-trait anxiety; in addition, the behavioral data did not show a PES effect, and, moreover, the diffusion model decomposition revealed that for both anxiety groups, errors were followed by an unexpected decrease in non-decision time and a decrease in discriminability (i.e., drift rate difference between targets and lures). Therefore, we feel the current study presents a more compelling case in favor of the increased response caution explanation of PES.

The present study shows that the drift diffusion model can be used not only to theorize about the causes of PES, but also to decompose the behavioral after-effects of an error into its constituent psychological processes. Such a decomposition is considerably more informative than the standard analysis of mean RT and accuracy, and we believe that future on PES can benefit from taking a similar approach.

\section{References}

Baayen, R. H., Piepenbrock, R., \& Van Rijn, H. (1993). The CELEX lexical data base on CD-ROM. Philadelphia: Linguistic Data Consortium.

Botvinick, M. M., Braver, T. S., Barch, D. M., Carter, C. S., \& Cohen, J. D. (2001). Conflict monitoring and cognitive control. Psychological Review, 108, 624-652.

Brewer, N., \& Smith, G. A. (1989). Developmental changes in processing speed: Influence of speed-accuracy regulation. Journal of Experimental Psychology, 118, 298-310.

Brown, S. D., \& Heathcote, A. J. (2008). The simplest complete model of choice reaction time: Linear ballistic accumulation. Cognitive Psychology, 57, 153-178.

Cohen, J. D., Botvinick, M., \& Carter, C. S. (2000). Anterior cingulate and prefrontal cortex: Who's in control? Nature Neuroscience, 3, 421-423.

Danielmeier, C., Eichele, T., Forstmann, B. U., Tittgemeyer, M., \& Ullsperger, M. (2011). Posterior Medial Frontal Cortex Activity Predicts Post-Error Adaptations in Task-Related Visual and Motor Areas. The Journal of Neuroscience, 31, 1780.

De Jong, R., Liang, C.-C., \& Lauber, E. (1994). Conditional and 



Figure 7. The three variability parameters of the diffusion model shown separately for post-correct and post-error trials. All variability parameters are slightly higher for post-error trials than for post-correct trials, but the error bars show that these effects are unreliable. Error bars represent standard errors of the mean.


Figure 8. For each of the three variability parameters of the diffusion model, box plots represent the distribution of the PES effect over participants. Comparison with the dashed horizontal line at zero suggests that PES effects on the variability parameters are not reliable. Boxes contain $50 \%$ of the values, whiskers enclose $80 \%$ of the values.

unconditional automaticity: A dual-process model of effects of spatial stimulus-response correspondence. Journal of Experimental Psychology: Human Perception and Performance, 20, 731-750.

Diederich, A., \& Busemeyer, J. (2006). Modeling the effects of payoff on response bias in a perceptual discrimination task: Boundchange, drift-rate-change, or two-stage-processing hypothesis. Perception \& Psychophysics, 86, 194-207.

Dutilh, G., Krypotos, A. M., \& Wagenmakers, E.-J. (in press). Task-related vs. stimulus-specific practice: A diffusion model account. Experimental Psychology.

Dutilh, G., Vandekerckhove, J., Tuerlinckx, F., \& Wagenmakers, E.-J. (2009). A diffusion model decomposition of the practice effect. Psychonomic Bulletin \& Review, 16, 1026-1036.

Edwards, W. (1965). Optimal strategies for seeking information: Models for statistics, choice reaction times, and human information processing. Journal of Mathematical Psychology, 2, 312329.

Fitts, P. M. (1966). Cognitive aspects of information processing. III. Set for speed versus accuracy. Journal of experimental psychology, 71, 849-857.

Forstmann, B. U., Dutilh, G., Brown, S. D., Neumann, J., Cramon, D. Y. von, Ridderinkhof, K. R., et al. (2008). Striatum and preSMA facilitate decision-making under time pressure. Proceed- ings of the National Academy of Sciences, 105, 17538.

Geddes, J., Ratcliff, R., Allerhand, M., Childers, R., Wright, R. J., Frier, B. M., et al. (in press). Modeling the effects of hypoglycemia on a two-choice task in adult humans. Neuropsychology.

Gold, J. I., \& Shadlen, M. N. (2007). The neural basis of decision making. Neuroscience, 30, 535-574.

Hajcak, G., McDonald, N., \& Simons, R. F. (2003). To err is autonomic: Error-related brain potentials, ANS activity, and posterror compensatory behavior. Psychophysiology, 40, 895-903.

Keuleers, E., \& Brysbaert, M. (2010). Wuggy: A multilingual pseudoword generator. Behavior Research Methods, 42, 627633.

Keuleers, E., Brysbaert, M., \& New, B. (2010). SUBTLEX-NL:A new measure for Dutch word frequency based on film subtitles. Behavior Research Methods, 42, 643-650.

Keuleers, E., Diependaele, K., \& Brysbaert, M. (2010). Practice effects in large-scale visual word recognition studies: A lexical decision study on 14,000 Dutch mono-and disyllabic words and nonwords. Frontiers in Psychology.

Klauer, K. C., Voss, A., Schmitz, F., \& Teige-Mocigemba, S. (2007). Process components of the implicit association test: A diffusion-model analysis. Journal of Personality and Social Psychology, 93, 353-368. 
Laming, D. (1968). Information theory of choice-reaction times.

Laming, D. (1979a). Autocorrelation of choice-reaction times. Acta Psychologica, 43, 381-412.

Laming, D. (1979b). Choice reaction preformance following an error. Acta Psychologica, 43, 199-224.

Li, R. C., Huang, C., Constable, R. T., \& Sinha, R. (2006). Imaging response inhibition in a stop-signal task: Neural correlates independent of signal monitoring and post-response processing. Journal of Neuroscience, 26, 186-192.

Luce, R. D. (1986). Response times. New York, NY: Oxford University Press.

Notebaert, W., Houtman, F., Opstal, F., Gevers, W., Fias, W., \& Verguts, T. (2009). Post-error slowing: An orienting account. Cognition, 111, 275-279.

Petrov, A. A., Horn, N. M., \& Ratcliff, R. (in press). Dissociable perceptual learning mechanisms revealed by diffusion-model analysis. Psychonomic Bulletin \& Review.

Pratte, M. S., Rouder, J. N., Morey, R. D., \& Feng, C. (2010). Exploring the differences in distributional properties between Stroop and Simon effects using delta plots. Attention, Perception, \& Psychophysics, 72, 2013-2025.

Rabbitt, P. (1966). How old and young subjects monitor and control responses for accuracy and speed. Journal of Experimental Psychology, 71, 264-272.

Rabbitt, P. (1979). How old and young subjects monitor and control responses for accuracy and speed. British Journal of Psychology, 70, 305-311.

Rabbitt, P., \& Rodgers, B. (1977). What does a man do after an error? An analysis of response programming. Quarterly Journal of Experimental Psychology, 29, 727-743.

Raftery, A. E. (1995). Bayesian model selection in social research. In P. V. Marsden (Ed.), Sociological methodology (pp. 111-196). Cambridge: Blackwells.

Ratcliff, R. (1978). A theory of memory retrieval. Psychological Review, 85, 59-108.

Ratcliff, R. (2002). A diffusion model account of response time and accuracy in a brightness discrimination task: Fitting real data and failing to fit fake but plausible data. Psychonomic Bulletin \& Review, 9, 278-291.

Ratcliff, R., Gomez, P., \& McKoon, G. (2004). A diffusion model account of the lexical decision task. Psychological Review, 111, $159-182$

Ratcliff, R., \& McKoon, G. (2008). The diffusion decision model: Theory and data for two-choice decision tasks. Neural Computation, 20, 873-922.

Ratcliff, R., \& Rouder, J. (1998). Modeling response times for two-choice decisions. Psychological Science, 9, 347-356.

Ratcliff, R., Schmiedek, F., \& McKoon, G. (2008). A diffusion model explanation of the worst performance rule for reaction time and IQ. Intelligence, 36, 10-17.

Ratcliff, R., Thapar, J., \& McKoon, G. (2001). The effects of aging on reaction time in a signal detection task. Psychology and Aging, 16, 323-341.

Ratcliff, R., Thapar, J., \& McKoon, G. (2006). Aging, practice, and perceptual tasks: A diffusion model analysis. Psychology and Aging, 21, 353-371.

Ratcliff, R., Thapar, J., \& McKoon, G. (2010). Individual differences, aging, and IQ in two-choice tasks. Cognitive psychology, $60,127-157$

Ratcliff, R., \& Van Dongen, H. P. A. (2009). Sleep deprivation affects multiple distinct cognitive processes. Psychonomic Bulletin \& Review, 16, 742-751.

Rouder, J. N., Speckman, P. L., Sun, D., Morey, R. D., \& Iverson,
G. (2009). Bayesian $t$ tests for accepting and rejecting the null hypothesis. Psychonomic Bulletin \& Review, 16, 225-237.

Schmiedek, F., Oberauer, K., Wilhelm, O., Suss, H.-M., \& Wittmann, W. W. (2007). Individual differences in components of reaction time distributions and their relations to working memory and intelligence. Journal of Experimental Psychology: General, 136, 414-429.

Schwarz, G. (1978). Estimating the dimension of a model. Annals of Statistics, 6, 461-464.

Shiels, K., \& Hawk, J. L. W. (2010). Self-regulation in ADHD: The role of error processing. Clinical Psychology Review, 951-961.

Smith, G. A., \& Brewer, N. (1995). Slowness and age: Speedaccuracy mechanisms. Psychology and Aging, 10, 238-247.

Speckman, P. L., Rouder, J. N., Morey, R. D., \& Pratte, M. S. (2008). Delta plots and coherent distribution ordering. The American Statistician, 62, 262-266.

Tops, M., \& Boksem, M. A. (2010). Cortisol involvement in mechanisms of behavioral inhibition. Psychophysiology, 1-10.

Van Ravenzwaaij, D., Brown, S., \& Wagenmakers, E.-J. (in press). An integrated perspective on the relation between response speed and intelligence. Cognition.

Van Ravenzwaaij, D., Van der Maas, H. L. J., \& Wagenmakers, E.-J. (in press). Does the name-race implicit association test measure racial prejudice?

Vandekerckhove, J., \& Tuerlinckx, F. (2007). Fitting the Ratcliff diffusion model to experimental data. Psychonomic Bulletin \& Review, 14, 1011-1026.

Vandekerckhove, J., \& Tuerlinckx, F. (2008). Diffusion model analysis with MATLAB: A DMAT primer. Behavior Research Methods, 40, 61-72.

Verguts, T., Notebaert, W., Kunde, W., \& Wühr, P. (2011). Postconflict slowing: Cognitive adaptation after conflict processing. Psychonomic Bulletin \& Review, 18, 76-82.

Vickers, D., \& Lee, M. D. (1998). Dynamic models of simple judgments: I. Properties of a self-regulating accumulator module. Nonlinear Dynamics, Psychology, and Life Sciences, 2, 169194.

Voss, A., Rothermund, K., \& Voss, J. (2004). Interpreting the parameters of the diffusion model: An empirical validation. Memory \& Cognition, 32, 1206-1220.

Wagenmakers, E.-J. (2009). Methodological and empirical developments for the Ratcliff diffusion model of response times and accuracy. European Journal of Cognitive Psychology, 21, 641671.

Wagenmakers, E.-J., Ratcliff, R., Gomez, P., \& McKoon, G. (2008). A diffusion model account of criterion shifts in the lexical decision task. Journal of Memory and Language, 58, 140-159.

Wagenmakers, E.-J., Van der Maas, H. L. J., \& Grasman, R. P. P. P. (2007). An EZ-diffusion model for response time and accuracy. Psychonomic Bulletin \& Review, 14, 3 .

Wetzels, R., Matzke, D., Lee, M. D., Rouder, J. N., Iverson, G. J., \& Wagenmakers, E.-J. (in press). Statistical evidence in experimental psychology: An empirical comparison using 855 t-tests. Perspectives on Psychological Science.

Wetzels, R., Raaijmakers, J. G. W., Jakab, E., \& Wagenmakers, E.J. (2009). How to quantify support for and against the null hypothesis: A flexible WinBUGS implementation of a default Bayesian t-test. Psychonomic bulletin \& review, 16, 752-760.

White, C. N., Ratcliff, R., Vasey, M., \& McKoon, G. (2009). Dysphoria and memory for emotional material: A diffusion-model analysis. Cognition \& emotion, 23, 181-205.

White, C. N., Ratcliff, R., Vasey, M. W., \& McKoon, G. (2010a). Anxiety enhances threat processing without competition among 
12 GILLES DUTILH $^{1}$, JOACHIM VANDEKERCKHOVE ${ }^{2}$, BIRTE U. FORSTMANN ${ }^{1}$, EMMANUEL KEULEERS ${ }^{3}$, MARC BRYSBAERT ${ }^{3}$, AND ERIC-JAN WAGENMAKERS ${ }^{1}$ multiple inputs: A diffusion model analysis. Emotion, 10, 662Using diffusion models to understand clinical disorders. Journal 677.

of Mathematical Psychology, 54, 39-52.

White, C. N., Ratcliff, R., Vasey, M. W., \& McKoon, G. (2010b). 